\title{
DESIGN AND DEVELOPMENT OF FALL DETECTOR USING FALL ACCELERATION
}

\author{
Sudarshan B G ${ }^{1}$, Raveendra Hegde ${ }^{2}$, Prasanna Kumar S C ${ }^{\mathbf{3}}$, Satyanarayana B S \\ ${ }^{1}$ Assistant Professor, ${ }^{3}$ Professor and Head, ${ }^{4}$ Principal, Dept. of Instrumentation Technology, RVCE, Bangalore, \\ Karnataka, India, \\ sudharshanbg@gmail.com,prasannakumar@rvce.edu.in,principal@rvce.edu.in
}

\begin{abstract}
Fall of patients and aged people may become fatal if unnoticed in time. The concept is to have a fall detection system which sends alarm to the concerned people or to the doctor, at the time of eventuality. To minimize fall and its related injuries continuous surveillance of subjects who are diseased and prone to fall is necessary. The article discusses the design and development of a prototype of an electronic gadget which is used to detect fall among elderly and the patients who are prone to it. In this article, the body posture is derived from change of acceleration in three axes, which is measured using triaxial accelerometer (adxl335). The sensor is placed on the lumbar region to study the tilt angle. The acceleration values in each axis are compared twice with threshold and also a delay of 20 secs between two comparisons, to reduce the false alarms. Values of the threshold voltage are selected by experimental methods. The algorithm is executed by microcontroller (PIC16F877A). The location of fall is determined by GPS receiver, which is programmed to track the subject continuously. On detection of fall, the device sends a text message through GSM modem, and communicates it to computer through ZigBee transceivers. The device can also be switched to only alarm if text message is not required. The prototype developed is tested on many subjects and also on volunteers who simulated fall. Out of 50 trials $96 \%$ of accuracy is achieved with zero false alarms for daily activities like jogging, skipping, walking on stairs, and picking up objects.
\end{abstract}

Index Terms: Fall Detector, Medical Alarming System, Personal Emergency Response System, triaxial accelerometer, microcontroller

\section{INTRODUCTION}

Fall is a clinical feature of many diseases such as Parkinson's disease, ectopic of heart, vestibulocochlear defects etc. Falls occur even at home and also in hospitals. Increase in number of patients results in difficulties of manual monitoring by the hospital staff, which leave the patients vulnerable to fall. With the advent of modernization and western culture, the nuclear families are on rise. This has led to single aged people living alone with geriatric problems. Falls are usually fatal due to head injuries and also because of not being treated in time. Falls not only cause physical, but also psychological trauma. People with history of fall are more prone to such attacks. The falls result in sustained psychological effects such as fear, increasing dependence [1]. Importance of preventing complications of fall lies in early detection and prevention of fall. According to survey done by Centres for Disease Control and Prevention (CDC), 33\% of aged people fall every year [2]. The old-age dependency ratio (the number of people 65 and over relative to those between 15 and 64) is projected to increase from value of $22 \%$ in 2010 to $37 \%$ by 2050 [3]. Fall related injuries are not only social burden but also economic aspect. Based on data from a survey done in US in the year 2000 , total annual estimated costs were between $\$ 16$ billion and $\$ 19$ billion for nonfatal fall-related injuries and approximately $\$ 170$ million dollars [4]. Fall-related death rates in the United States increased between 1999 and 2004, from 29 to 41 per 100,000 population [4]. In Indian population such a statistics is not available. A low cost personal emergency response system can also be used by fireman and mountaineers who are at the risk of fall and its related injuries. With the above mentioned factors such as medical and economic issues, lies the importance in design and development of a fall detector.

Efforts to detect fall among elderly is being done over several years. Detailed literature review gives us four types of approaches to detect fall, namely: wearable sensor, ambient sensor, combination of wearable and ambient sensor and image processing. The body wearable method is found economical and suitable for both indoor and outdoor scenarios. S.Y.Sim et al. [1] tried to place accelerometer in shoe and experiments shown that the algorithm is sensitive $(81.5 \%)$ when the sensor is placed in tongue of shoe. WenChang Cheng et al. [5] used chest or waist worn triaxial accelerometer to derive body posture. Cascade Ada-Boost support vector machine is employed to classify fall from other activities with high accuracy (more than 98\%). A new approach by placing the sensors on garment is done by Khalil 
Niazmand et al. [6]; it can be worn without any discomfort. The algorithm achieved sensitivity of $97.5 \%$ and specificity of $96.92 \%$. The combination of accelerometer and gyroscope is placed on thigh and chest region and the algorithm employed succeeded to give $92 \%$ accuracy [7]. A new algorithm was proposed by Ravi Narasimhan [8]. Data is acquired by triaxial accelerometer and threshold window is computed by experimental values. The specificity of $100 \%$ and sensitivity of $99 \%$ was achieved by placing the accelerometer on torso and by applying algorithm. Many researchers worked to develop fall detecting system using built-in tri-axial accelerometer of mobile phone. Frank Sposaro et al.[9] developed an application for android phone. It asks the user to communicate when a fall is detected, messages are sent to social contacts if not replied. The emergency alarm is raised if both fail. Yi He et al. [10] considered smart phone as a waist worn device and developed an algorithm to send MMS (multimedia message service) to pre-selected contacts and the location is determined by GPS coordinate and Google map.

The proposed method in this article is to detect falls which include only change of plane of human body due to various reasons such as geriatric problems and its associated diseases like Parkinson's disease, ectopics of heart, vestibulocochlear defects etc.Derived human body posture is compared to predefined threshold values to separate fall from daily activities. Repeated readings of sensor response for different tilt angles give threshold values. Since, change of plane of body and sudden changes in acceleration are involved in fall; triaxial accelerometer is a suitable sensor. The changes of acceleration in 3 axes are monitored continuously. False alarms are reduced by deriving posture twice separated by 20 second delay.

The section 2 of the paper describes methodology to detect fall. Selection of threshold voltage is discussed in section 3 . The suitable anatomical position to place the device is explained in section 4. Results of experiments are given in section 5. The paper is concluded in the section 6 .

\section{METHODOLOGY TO DETECT FALL}

The technique of detecting fall relies on deriving human body posture with a suitable sensor placed at appropriate anatomical position and an effective algorithm which precisely distinguish daily activities and fall. Since, change of plane of body and sudden changes in acceleration are involved in fall; triaxial accelerometer is a suitable sensor. The changes of acceleration in 3 axes are monitored continuously. When the changes in acceleration fall in the window of threshold values, it is decided as fall.

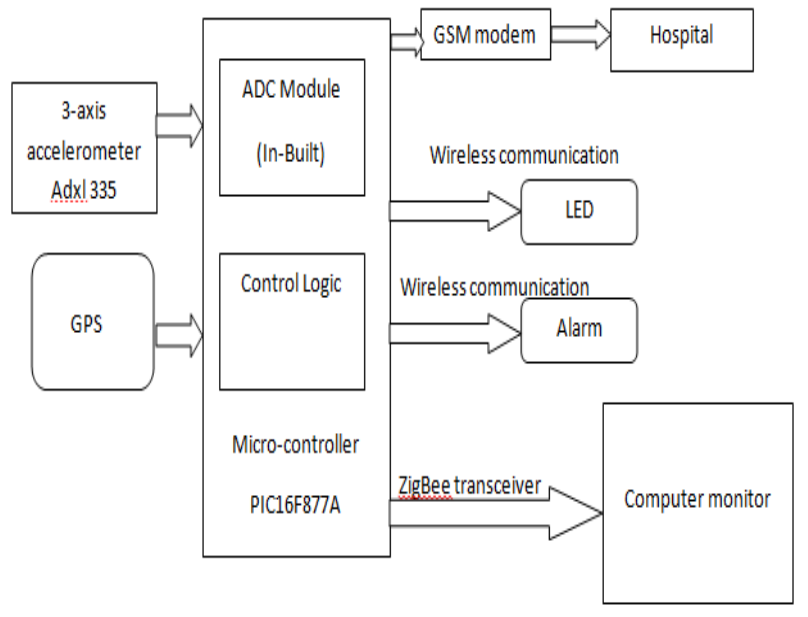

Fig -1: Block Diagram of fall detector

The block diagram (Fig -1) possesses four main sections, input, the controller, communication protocols and output devices. The input consists of acceleration values and GPS data. The transmitter pin of GPS is connected to the receiver (UART) of microcontroller. Location data is read serially byte by byte which gives the information of longitude and latitude. The triaxial accelerometer sensor $(\operatorname{adxl335})$ is used to derive body posture of the subject. Acceleration and angle information of three axes is produced as three analog signals which vary with body posture. Acceleration value generated in each axis is read through separate pins, selecting one analog input at a time. Analog signals generated by the sensor are digitized by analog to digital converter of the microcontroller (PIC16F877A). The digitized values are compared to predefined threshold values. Human body posture is derived twice; between two posture readings a delay of 20 seconds is introduced. The delay time helps to reduce false alarms.

ZigBee transceiver pair (Tarang F4) is used for communication between the microcontroller and the computer and indicators. An alert message is sent to a hospital phone number when a fall is detected. The GSM modem used is SIM 300 V_7.03. ZigBee transceiver and GSM modem are connected to RS232 port of microcontroller board via a switch. The system can be switched send either SMS alert or just a ZigBee alert to the indicators (LED and beeper). This facility avoids sending unnecessary SMS while patient is at home with family members.

The microcontroller board, the ZigBee transceiver and the GSM modem are powered up by a single DC source of $12 \mathrm{~V}$, $2 \mathrm{~A}$. The sensor board and GPS device use $5 \mathrm{~V}$ generated by built in voltage regulator of microcontroller board. 


\subsection{Flow Chart for the Algorithm}

The Fig -2 depicts the detailed flow of program. The microcontroller is programmed to monitor and track the subject continuously. The initialization part includes configuring analog to digital converter and Universal Asynchronous Synchronous Receiver Transmitter (UART). To remove false alarms induced by fall like activities the acceleration values are measured and compared two times. Between two measurements and comparisons, a delay of 20 seconds is introduced. The flow chart shows two modes of operation, mode1: send text message to pre-stored phone numbers, mode2: send only alarming signal to the indicators. Mode 2 is used when the patient is alone and the device is switched to mode 1 when family members are around.

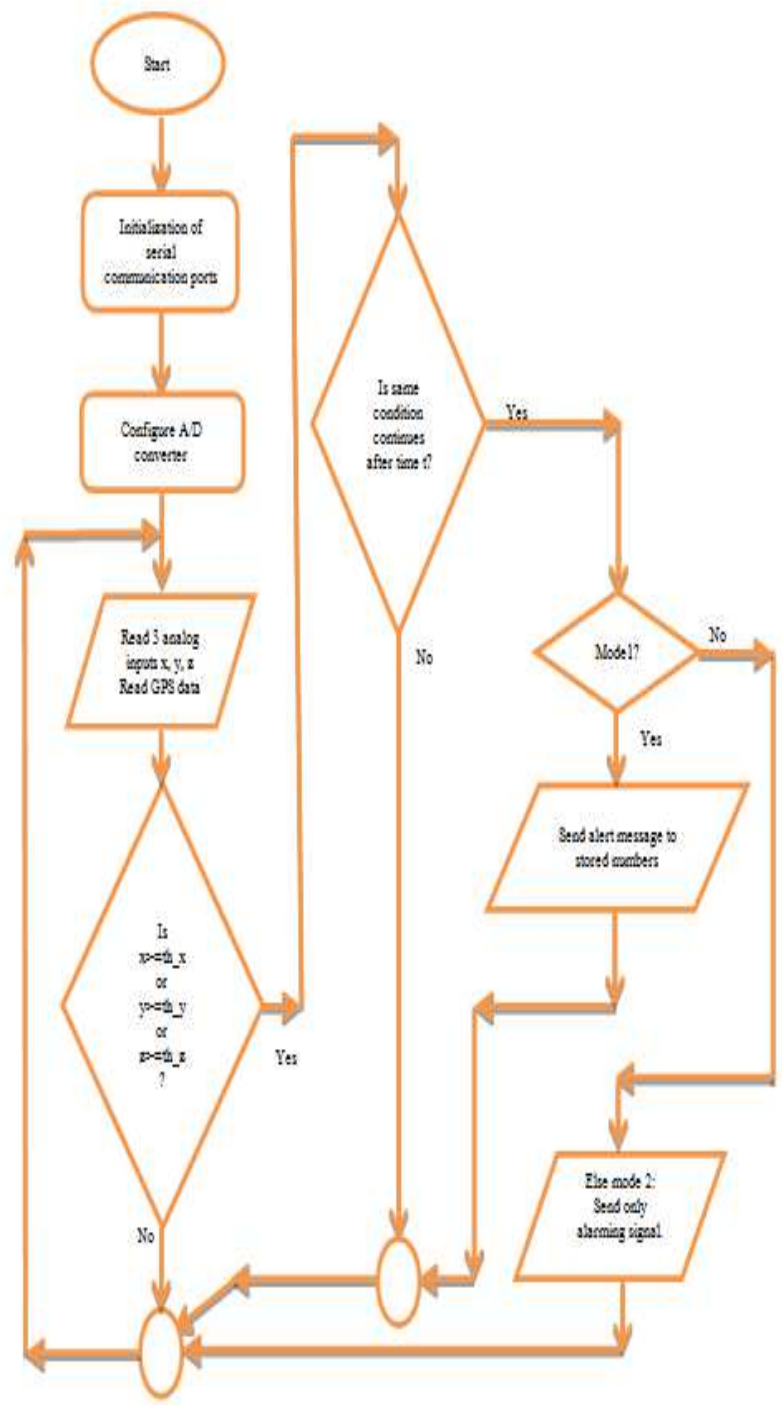

Fig -2: Flow chart for implemented algorithm

\subsection{Algorithm}

Step1. Initialize serial communication ports of microcontroller Step2. Configure ADC and analog input channel Step3. Initialize GPS and GSM modules.

Step4. Receive analog inputs from sensor.

Step5. Receive location information from GPS.

Step6. Compare the digital values of sensor signal with predefined thresholds. If acceleration is greater than the threshold go to step7, else go to step4.

Step7. Wait for time $\mathrm{t}$ and again read acceleration values. Compare with same threshold again.

Step8. Is fall detected? If yes go to step9, go to step4 if not. Step9. Send text message to stored numbers, send alarming signal to indicators if the operating mode1. Send only alarming signals to the indicators if mode2.

\section{SELECTION OF THRESHOLD VOLTAGE}

The threshold voltage is selected by readings of sensor responses for different tilted positions. Fig -3 shows sensor outputs for different fall scenarios. It is observed that for different types of falls.
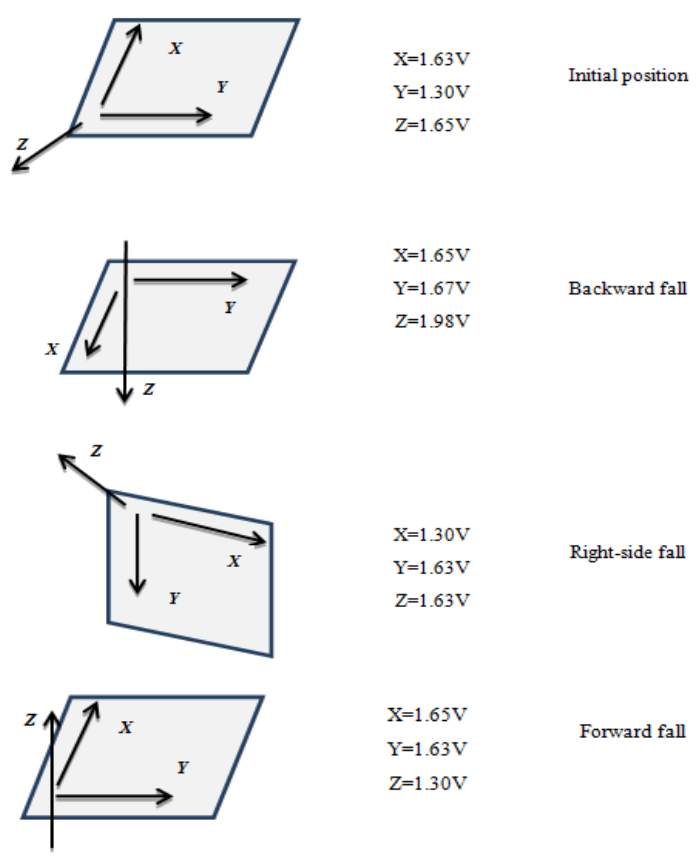

Forward fall

$\mathrm{Y}=1.63 \mathrm{~V}$

$=1.30 \mathrm{~V}$

$\mathrm{X}=1.96 \mathrm{~V}$

$\mathrm{Y}=1.64 \mathrm{~V}$

Left-side fall 
Backward fall is indicated by $\mathrm{Z}$ axis, forward and right side fall is indicated by $\mathrm{Y}$ axis values, and $\mathrm{Z}$ axis indicates left side fall. From the observation of sensor responses, $1.96 \mathrm{~V}, 1.63 \mathrm{~V}$, $1.98 \mathrm{~V}$ are considered as threshold values for $\mathrm{X}, \mathrm{Y}, \mathrm{Z}$ axes respectively.

\section{PLACEMENT OF THE DEVICE ON HUMAN BODY}

Anatomical position of the device influences the accuracy and specificity to a great extent. If the detection algorithm depends mainly on the body posture and tilt, then torso is more suitable place [11]. Stefano Abbate et al. [11] listed different possible anatomical positions to derive various postures (table 1).

Table -1: Different positions of sensor corresponding derived posture

\begin{tabular}{|l|l|}
\hline Sensor position & Identified posture \\
\hline Chest & (standing or sitting), (bending or lying) \\
\hline Waist & (bending or standing or sitting), (lying) \\
\hline Chest + Thigh & bending, lying, standing, sitting \\
\hline
\end{tabular}

Since algorithm of the project depends on thresholds of tilt, it is suitable to select waist portion to place the device. If the device is placed just below stomach, obesity of the subject may induce some tilt. To avoid this, lumbar vertebrae is appropriate position to place the device as shown in the Fig -4.

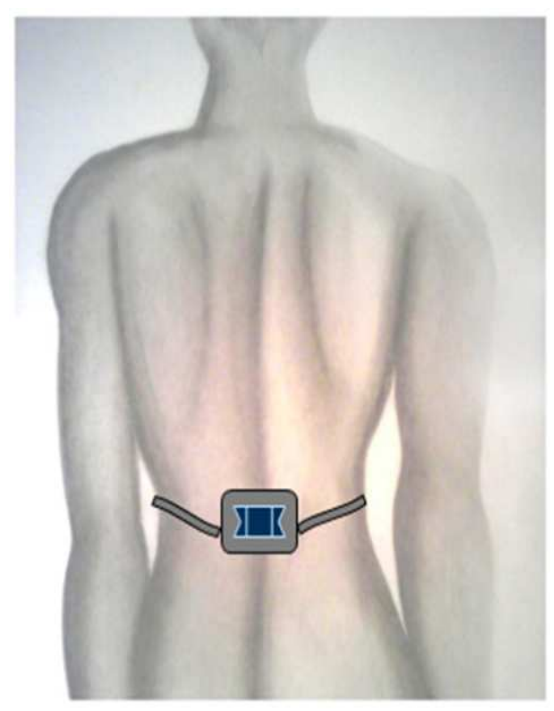

Fig -4: Placement of the device.

\section{RESULTS AND DISCUSSIONS}

The components are enclosed in a plastic box and tied in the waist region as shown in Fig -5. The prototype is tested on five volunteers (healthy adult males) in the PG research lab and RVCE health center. Each subject is made to fall on bench and bed, and many fall like activities (skipping, jogging, picking objects from the floor and walking on staircase). The following figure shows one of the subjects simulating fall.

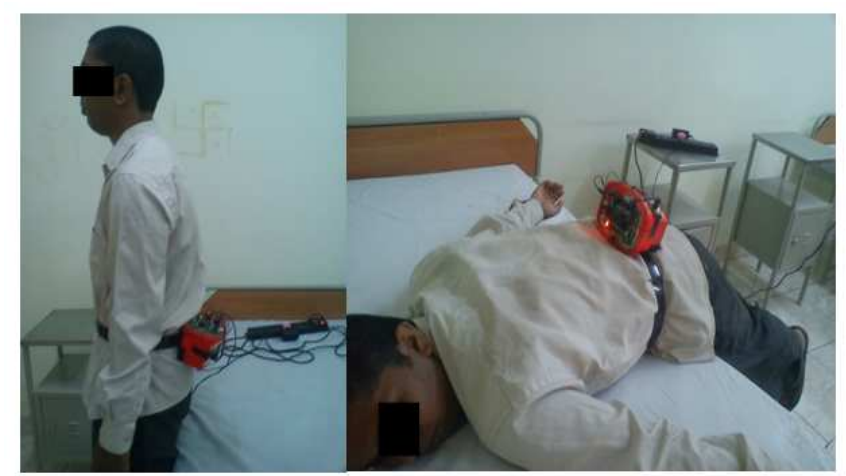

Fig -5: One of the volunteers simulating fall

Out of fifty trials (ten trials each subject) only two events are not detected. The trials included forward fall, falling sideways and backward fall. The missed detection happened when the subjects knelt down slowly and leaned forward without giving any jerk. Since most of the falls not likely to happen this way, the missed detections do not have significance. The fall simulating activities like jogging, skipping, walking on stairs and picking objects did not create any false alarms. The outcome of the experiments is listed in the table 2. As we see from the table the accuracy is $96 \%$.

Table -2: Analysis of prototype testing

\begin{tabular}{|l|l|l|l|}
\hline Subject & $\begin{array}{l}\text { Number of } \\
\text { trials }\end{array}$ & $\begin{array}{l}\text { Number of } \\
\text { detections }\end{array}$ & $\begin{array}{l}\text { Number of } \\
\text { false alarms }\end{array}$ \\
\hline 1 & 10 & 9 & 0 \\
\hline 2 & 10 & 10 & 0 \\
\hline 3 & 10 & 10 & 0 \\
\hline 4 & 10 & 9 & 0 \\
\hline 5 & 10 & 10 & 0 \\
\hline
\end{tabular}

The snapshots of assembly of components are shown in the Fig -6 . 


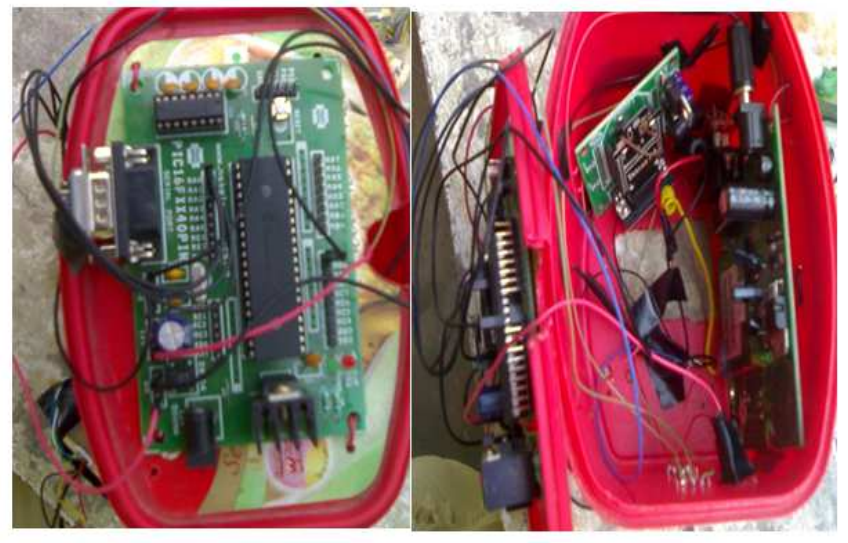

Fig-6: Assembly of components

\section{CONCLUSIONS}

In this research paper, we had achieved our primary goal of creating a working prototype able to recognize both dangerous posture and falls from non-falls, with wireless communication to the indicators and computer. Looking at the underlying detection process, our fall detection system improves on previous systems and designs by giving zero false alarms, bearing low cost, and with new anatomical position for the sensor. We incorporated hybrid fall detection algorithm derived from existing algorithms, and interfaced the GPS receiver successfully to locate fall. The accuracy is $96 \%$.

\section{REFERENCES}

[1] S.Y.Sim, H.S.Jeon, G.S.Chung, S.K.Kim, S.J.Kwon, W.K.Lee, K.S.Park, 'Fall detection algorithm for the elderly using acceleration sensors on the shoes'. 33rd Annual International Conference of the IEEE EMBS Boston, Massachusetts USA, August 30 - September 3, 2011. pp 4935-4938.

[2] GohYongli, Ooi Shih Yin and Pang Ying Han, 'State of the Art: A Study on Fall Detection', World Academy of Science, Engineering and Technology 62, pp 294-298, 2012.

[3] KorayOzcan, AnvithKatteMahabalagiri, Mauricio Casares, Member, and SenemVelipasalar, 'Automatic Fall Detection and Activity Classification by a Wearable Embedded Smart Camera', IEEE Journal on Emerging and Selected Topics in Circuits and Systems, VOL. 3, NO. 2, JUNE 2013. Pp 125-137.

[4] Leanne Currie, 'Patient Safety and Quality: An Evidence-Based Handbook for Nurses', chapter 10. Fall and Injury Prevention. AHRQ Publication No. 08-0043.

[5] Wen-Chang Cheng and Ding-Mao Jhan, 'Triaxial Accelerometer-Based Fall DetectionMethod Using a Self-Constructing Cascade-AdaBoost-SVM Classifier', IEEE Journal of Biomedical and Health Informatics, VOL. 17, NO. 2, MARCH 2013, pp 410-419.
[6] Khalil Niazmand, Claudius Jehle, Lorenzo T. D Angelo and Tim C. Lueth, 'A New Washable Low-Cost Garment for Everyday Fall Detection', 32nd Annual International Conference of the IEEE EMBS Buenos Aires, Argentina, August 31 - September 4, 2010. PP 6377-6380.

[7] Qiang Li, John A. Stankovic, Mark Hanson, Adam Barth, 'Accurate, Fast Fall Detection Using Gyroscopes and Accelerometer-Derived Posture Information'Wearable and Implantable Body Sensor Networks,. BSN 2009. Sixth International Workshop, 2009 Digital Object Identifier: 10.1109/BSN. Pp 138143.

[8] Ravi Narasimhan, 'Skin-Contact Sensor for Automatic Fall Detection', 34th Annual International Conference of the IEEE EMBS San Diego, California USA, 28 August - 1 September,2012. pp 4038-4041.

[9] Frank Sposaro and Gary Tyson, 'i Fall: An android Application for Fall Monitoring and Response', 31st Annual International Conference of the IEEE EMBS Minneapolis, Minnesota, USA, September 2-6, 2009. Pp 6119-6122.

[10] Yi He, Ye Li, and Shu-OiBao, 'Fall Detection by BuiltIn Tri-Accelerometer of Smartphone', Proceedings of the IEEE-EMBS International Conference on Biomedical and Health Informatics (BHI 2012) Hong Kong and Shenzhen, China, 2-7 Jan 2012. Pp 184-187.

[11] Stefano Abbate , MrcoAvvenuti , Guglielmo Cola , Paolo Cors "Recognition of false alarms in fall detection systems" 30th Annual International IEEE EMBS Conference Vancouver, British Columbia, Canada, August 20-24, 2008. pp 234-239. 